\title{
Studying Membrane Trafficking in Toxoplasma gondii Using Correlative Light and Electron Microscopy (CLEM)
}

Ru-ching Hsia ${ }^{1,2}$, John Strong ${ }^{1}$, Julia Romano ${ }^{3}$ and Isabelle Coppens ${ }^{3}$

1. Electron Microscopy Core Imaging Facility, University of Maryland Baltimore, Baltimore, USA

2. Department of Neural and Pain Sciences, University of Maryland Dental School, Baltimore, USA

3. Department of Molecular Microbiology and Immunology, Johns Hopkins University, Baltimore, USA

Toxoplasma gondii is an obligate intracellular parasite that can cause fatal encephalitis in immunocompromised individuals. During infection, T. gondii multiplies in the cytoplasm of mammalian cells within a self-made membrane-bound compartment - the parasitophorous vacuole (PV) from which it acquires nutrients from the mammalian host. The PV membrane has a unique lipid and protein composition and does not fuse with endocytic or exocytic organelles in the host cytoplasm. Previous studies have shown that Toxoplasma acquires cholesterol and sphingolipids from their mammalian host [1]. It has been suggested that Toxoplasma targets several mammalian Rab GTPases, intercepts cellular traffic mediated by Rab recycling vesicles and Rab vesicles from the secretory pathway, and acquires nutrients such as lipids by macro-endocytosis of nutrient-filled vesicles into the PV. In this study, we examined the ultrastructure of host GFP-Rab vesicles located near and within the PV using correlative light and electron microscopy (CLEM).

HeLa cells transfected with GFP-tagged Rab GTPase and infected with T. gondii (expressing RFP) were grown on $10 \mathrm{~mm}$ glass coverslips with a locator grid pattern generated with a carbon coater. Cells containing GFP-tagged Rab vesicles and RFP-tagged Toxoplasma were identified by fluorescence microscopy and their locations were recorded according to the locator pattern. Serial optical section images of several cells of interest were collected using a Nikon Eclipse E800 microscope. After light microscopy (LM) imaging, cells were fixed, dehydrated, and embedded in epoxy resin directly on the coverslips. The cells of interest were located in the resin under the dissecting microscope. Serial ultrathin sections were collected on slot grids and imaged in a transmission electron microscope (TEM) at $80 \mathrm{keV}$ (Tecnai T12, FEI). Photoshop CS2 and Image J were used to analyze the LM and EM images.

A Toxoplasma-infected HeLa cell expressing GFP-Rab was selected for further CLEM analysis (Figure 1a). Two T. gondii PVs (PV-a and PV-b) were present in the cell with punctuate GFP-Rab signal (white arrows) closely associated with or trapped within the PVs (Figure 2). We identified five GFP-Rab positive targets and further analyzed their ultrastructure and temporal and spatial relationship with the parasites and PV by EM. Approximately 60 Serial sections (70 nm thickness) were collected and analyzed by TEM to identify the specific sections corresponding to the LM optical sections. A group of 5 serial sections correlating to one of the GFP-Rab positive area is presented in Figure 3.

We describe here a CLEM workflow appropriate for the study of membrane trafficking in Toxoplasma gondii-infected cells. Our results confirmed that GFP-Rab positive LM signals observed adjacent to the Toxoplasma PV consist of double membrane vesicles. These vesicles are most likely Rab-GFP positive vesicles enclosed within the Toxoplasma PV membrane confirming our hypothesis that host nutrientfilled vesicles are taken up by the parasite via a process similar to phagocytosis. 
References:

[1] Coppens, I., Sinai, A.P., Joiner, K.A. J. Cell Biol 149 (2000), p. 167.
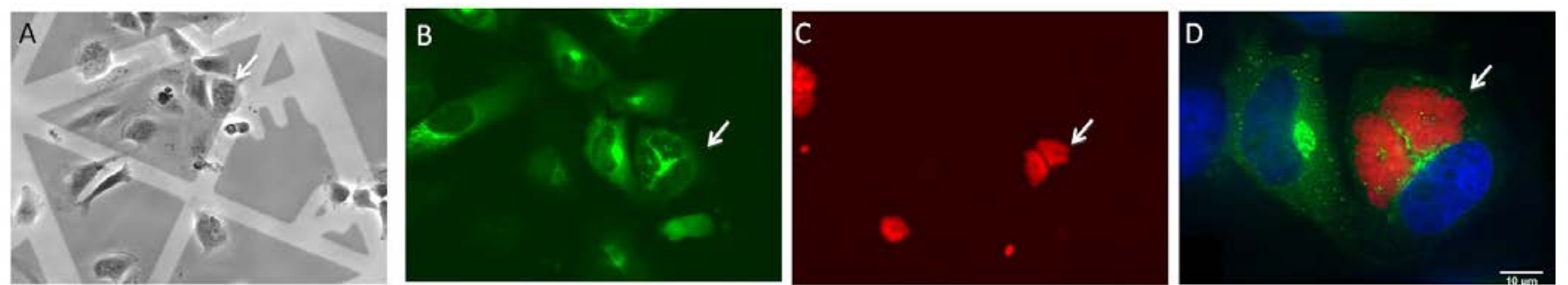

Figure 1. Localization of T. gondii-infected Hela cell that also expresses GFP-Rab by light microscopy. Bright field LM images showing a cell of interest and the locator grid pattern at 200X (A) magnification. Fluorescence images of one cell (white arrow) located in E4 region containing two Toxoplasma PVs was chosen for CLEM analysis (B and C). One optical section LM image is shown in D.
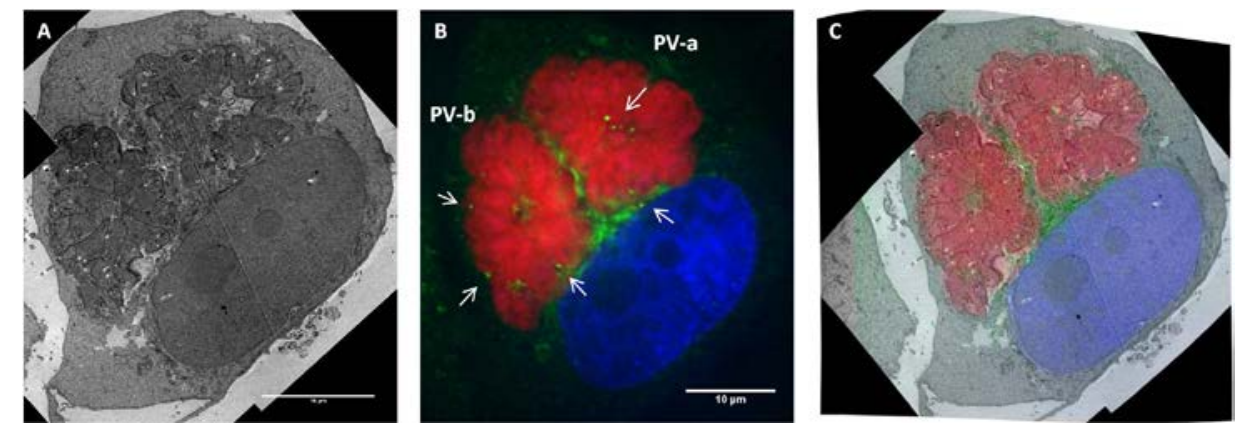

Figure 2. Correlation of the TEM image (A) with one of the optical section LM image of the same $T$. gondii infected cell. White arrows indicate host Rab GTPase vesicles trapped near or within PV (B). Overlay of LM and EM images reveals the ultrastructure of the corresponding LM signals (C)
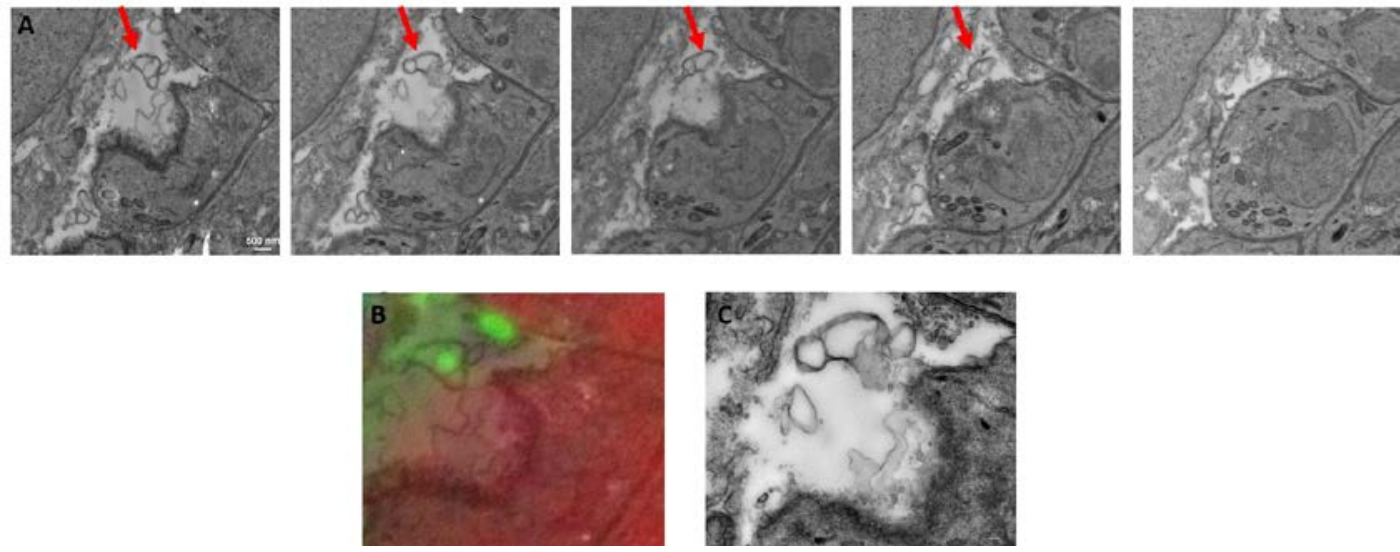

Figure 3. TEM images confirm that the ultrastructure of the vesicles correlate to GFP-Rab signal in PV-b by LM. Five serial sections (A) illustrate the temporal and spatial relationship of the vesicles, the parasite and the PV. GFP-Rab signal in LM is overlayed on the TEM image (B) to reveal the vesicle ultrastructure and the neighboring parasite $(\mathrm{C})$. 